\title{
Arbitrary Force-Constant Changes in the Crystal Impurity Problem
}

\author{
Philip D. Mannheim* \\ Department of Physics, University of Connecticut, Storrs, CT 06269
}

(December 8, 2005)

\begin{abstract}
In a previous study of the dynamics of crystals with substitutional point defects we had obtained simple and exact expressions for the positions of the perturbed crystal modes and the intensities in them in the case in which both the host-host and the host-impurity force-constants were taken to be central and nearest neighbor. Such expressions required knowledge of only one pure crystal lattice Green's function, the one at the defect site itself. In this paper we extend our previous study to incorporate non-central force-constants as well. We find that the same simple expressions which we had previously obtained in the nearest neighbor central force-constant case also hold without any modification at all in the case of isotropic force-constant changes in crystals whose host-host force-constants are isotropic, and in the particular mixed case in which the fractional changes in the central and isotropic components of the force-constants are equal to each other. In the most general arbitrary nearest neighbor force-constant case we obtain a reasonably compact exact expression for the dynamics of the perturbed modes which only involves a total of two pure crystal lattice Green's functions.
\end{abstract}

\section{INTRODUCTION}

Studies of nuclear resonant inelastic x-ray scattering made possible by the advent of dedicated synchrotron rings have generated renewed interest in the crystal impurity problem (see e.g. [1-3]). In the typical experimental set-up x-rays are inelastically scattered off Mössbauer active nuclei embedded in host materials such as crystals. Such Mössbauer active nuclei act as impurities in the otherwise perfect host crystals into which they are inserted, and lead to a modification of the crystal dynamics of the host, with it being the response of the perturbed system rather than that of the host itself which is then measured in the inelastic x-ray scattering process. The general theory for such impurity-induced modifications can for instance be found in [4] where exact, analytic expressions for the effect of a mass

\footnotetext{
*Email address: philip.mannheim@uconn.edu
} 
change at the defect site are given. For the case of defect-induced force-constant changes equally exact, analytic expressions have been given $[5,6]$ in the particular case where both the host-host and host-defect force-constants are central and nearest neighbor. Specifically, for such a situation it was found in the physically interesting central force-constant bodycentered and face-centered cubic crystal host cases that for both of them the positions of the frequencies of the impure crystal were given as the solutions to

$$
1-\rho\left(\omega^{2}\right) S\left(\omega^{2}\right)=0
$$

where $\rho\left(\omega^{2}\right)$ is given by

$$
\rho\left(\omega^{2}\right)=\frac{M}{M^{\prime}}-1+\frac{M \omega^{2}}{A_{x x}(0,0)}\left(1-\frac{A_{x x}(0,0)}{A_{x x}^{\prime}(0,0)}\right)=\frac{M}{M^{\prime}}-1+\frac{2 \omega^{2}}{\omega_{\max }^{2}}\left(1-\frac{A_{x x}(0,0)}{A_{x x}^{\prime}(0,0)}\right)
$$

$M$ and $M^{\prime}$ are the host and defect atom masses, $A_{x x}(0,0)$ and $A_{x x}^{\prime}(0,0)$ are the pure and impure self force-constants at the defect site, and the function $S\left(\omega^{2}\right)$ is given by

$$
S\left(\omega^{2}\right)=-1-M \omega^{2} g_{0}=\int_{0}^{\omega_{\max }^{2}} d \omega^{\prime 2} \frac{\omega^{\prime 2} \nu\left(\omega^{\prime 2}\right)}{\left(\omega^{2}-\omega^{\prime 2}\right)}
$$

as integrated over the density of squared eigenfrequencies of the pure crystal [7]. Additionally, the amplitude of vibration of the defect as defined via the impure crystal defect displacement normal mode expansion $u_{\alpha}(t)=(\hbar / 2 \omega)^{1 / 2} \sum_{\omega} \chi_{\alpha}\left(0, \omega^{2}\right)\left[a_{\omega} e^{-i \omega t}+a_{\omega}^{\dagger} e^{i \omega t}\right](\alpha=x, y, z)$ was found to be given by

$$
\left|\chi^{2}\left(0, \omega^{2}\right)\right|=\sum_{\alpha}\left|\chi_{\alpha}^{2}\left(0, \omega^{2}\right)\right|=\frac{1}{M N}\left(\frac{M}{M^{\prime}}\right)^{2}\left[\frac{1}{\left[1-\rho\left(\omega^{2}\right) S_{P}\left(\omega^{2}\right)\right]^{2}+\left[\pi \omega^{2} \nu\left(\omega^{2}\right) \rho\left(\omega^{2}\right)\right]^{2}}\right]
$$

for in-band modes with $\omega^{2} \leq \omega_{\max }^{2}\left(S_{P}\left(\omega^{2}\right)\right.$ is the principal value of $\left.S\left(\omega^{2}\right)\right)$, while being given by

$$
\left|\chi^{2}\left(0, \omega_{L}^{2}\right)\right|=\frac{1}{M}\left(\frac{M}{M^{\prime}}\right)^{2}\left[\frac{1}{\rho^{2}\left(\omega_{L}^{2}\right) T\left(\omega_{L}^{2}\right)+M / M^{\prime}-\left[1+\rho\left(\omega_{L}^{2}\right)\right]^{2}}\right]
$$

for localized modes which obey $1-\rho\left(\omega_{L}^{2}\right) S\left(\omega_{L}^{2}\right)=0$ with frequencies $\omega_{L}^{2}$ outside the band, with the function $T\left(\omega_{L}^{2}\right)$ being given by

$$
T\left(\omega_{L}^{2}\right)=\omega_{L}^{4} \int_{0}^{\omega_{\max }^{2}} d \omega^{\prime 2} \frac{\nu\left(\omega^{\prime 2}\right)}{\left(\omega_{L}^{2}-\omega^{\prime 2}\right)^{2}}
$$

For inelastic Mössbauer studies it is the quantity $\left|\chi^{2}\left(0, \omega^{2}\right)\right|$ which then gives the probability for nuclear resonant inelastic x-ray scattering at frequency $\omega$, with the experimentally measurable PDOS (the so-called partial density of states) at energy $E$ discussed for instance in [2] being given as $D(E)=M^{\prime}\left|\chi^{2}\left(0, \omega^{2}\right)\right| \nu(\omega)$ [8]. In the present paper we extend the analysis of $[5,6]$ to incorporate non-central nearest neighbor force-constants as well, an occurrence which is also of experimental concern. 


\section{SETTING UP THE FORCE-CONSTANT CHANGE PROBLEM}

In the standard harmonic approximation the equations of motion for the displacements from equilibrium $e^{-i \omega t} u_{\alpha}(\ell)$ of the atoms of a pure $3 \mathrm{~N}$ degree of freedom crystal lattice are given by

$$
\sum_{\beta, \ell^{\prime}}\left[A_{\alpha \beta}\left(\ell, \ell^{\prime}\right)-w^{2} M\left(\ell^{\prime}\right) \delta_{\alpha \beta} \delta\left(\ell, \ell^{\prime}\right)\right] u_{\beta}\left(\ell^{\prime}\right)=0
$$

where $\ell$ ranges from 0 to $N-1, \alpha=x, y, z, M(\ell)=M$ is the mass of the atom at site $\ell$, and $A_{\alpha \beta}\left(\ell, \ell^{\prime}\right)$ are the pure crystal force-constants. Similarly, for a system with a substitutional point impurity of mass $M^{\prime}$ located at the origin of coordinates and changed force-constants $A_{\alpha \beta}^{\prime}\left(\ell, \ell^{\prime}\right)$, the displacements from equilibrium are given as $e^{-i \omega^{\prime} t} u_{\alpha}(\ell)$, with Eq. (7) being replaced by

$$
\sum_{\beta, \ell^{\prime}}\left[A_{\alpha \beta}\left(\ell, \ell^{\prime}\right)-w^{\prime 2} M \delta_{\alpha \beta} \delta\left(\ell, \ell^{\prime}\right)\right] u_{\beta}\left(\ell^{\prime}\right)=\sum_{\beta, \ell^{\prime}} V_{\alpha \beta}\left(\ell, \ell^{\prime}\right) u_{\beta}\left(\ell^{\prime}\right)
$$

with the changes from the pure crystal case having been isolated in the perturbation

$$
V_{\alpha \beta}\left(\ell, \ell^{\prime}\right)=-w^{\prime 2}\left(M-M^{\prime}\right) \delta_{\alpha \beta} \delta(\ell, 0) \delta\left(\ell^{\prime}, 0\right)+A_{\alpha \beta}\left(\ell, \ell^{\prime}\right)-A_{\alpha \beta}^{\prime}\left(\ell, \ell^{\prime}\right)
$$

A formal solution for the positions of the frequency modes which satisfy Eq. (8) can be obtained in terms of the pure crystal lattice Green's functions as evaluated in the lattice site representation. Specifically, one first introduces the dynamical matrix of the pure crystal

$$
D_{\alpha \beta}(\overrightarrow{\mathbf{k}})=\frac{1}{M} \sum_{\ell} A_{\alpha \beta}(0, \ell) e^{-i \overrightarrow{\mathbf{k}} \cdot \overrightarrow{\mathbf{R}}(\ell)}
$$

as expressed in terms of the phonon modes $\overrightarrow{\mathbf{k}}$ of the translational invariant pure crystal, and then defines its eigenvectors and eigenvalues according to

$$
\begin{aligned}
& \sum_{\beta} D_{\alpha \beta}(\overrightarrow{\mathbf{k}}) \sigma_{\beta}^{j}(\overrightarrow{\mathbf{k}})=\omega_{j}^{2}(\overrightarrow{\mathbf{k}}) \sigma_{\alpha}^{j}(\overrightarrow{\mathbf{k}}), \\
& \sum_{\alpha} \sigma_{\alpha}^{* j}(\overrightarrow{\mathbf{k}}) \sigma_{\alpha}^{j^{\prime}}(\overrightarrow{\mathbf{k}})=\delta_{j j^{\prime}}, \quad \sum_{j} \sigma_{\alpha}^{* j}(\overrightarrow{\mathbf{k}}) \sigma_{\beta}^{j}(\overrightarrow{\mathbf{k}})=\delta_{\alpha \beta},
\end{aligned}
$$

and uses them to construct the pure crystal lattice Green's functions according to

$$
g_{\alpha \beta}\left(\omega ; \ell, \ell^{\prime}\right)=\frac{1}{N M} \sum_{\overrightarrow{\mathbf{k}}, j} \frac{\sigma_{\alpha}^{* j}(\overrightarrow{\mathbf{k}}) \sigma_{\beta}^{j}(\overrightarrow{\mathbf{k}}) e^{i \overrightarrow{\mathbf{k}} \cdot\left[\overrightarrow{\mathbf{R}}\left(\ell^{\prime}\right)-\overrightarrow{\mathbf{R}}(\ell)\right]}}{\left[\omega_{j}^{2}(\overrightarrow{\mathbf{k}})-\omega^{2}\right]}
$$

as summed over the three polarizations $j=(1,2,3)$ and $N$ momentum vectors $\overrightarrow{\mathbf{k}}$ of all the modes in the Brillouin zone. As constructed these Green's functions obey

$$
\sum_{\ell, \beta} A_{\alpha \beta}(0, \ell) g_{\alpha^{\prime} \beta}\left(\omega ; \ell, \ell^{\prime}\right)=M \omega^{2} g_{\alpha^{\prime} \alpha}\left(\omega ; 0, \ell^{\prime}\right)+\frac{\delta_{\alpha, \alpha^{\prime}}}{N} \sum_{\overrightarrow{\mathbf{k}}} e^{i \overrightarrow{\mathbf{k}} \cdot \overrightarrow{\mathbf{R}}\left(\ell^{\prime}\right)}
$$

and thus immediately allow us to solve Eq. (8) in the form 


$$
u_{\alpha}(\ell)=\sum_{\ell^{\prime}, \ell^{\prime \prime}, \beta, \gamma} g_{\alpha \beta}\left(\omega^{\prime} ; \ell, \ell^{\prime}\right) V_{\beta \gamma}\left(\ell^{\prime}, \ell^{\prime \prime}\right) u_{\gamma}\left(\ell^{\prime \prime}\right)
$$

with the eigenmodes then being given as the solutions to the (3N-dimensional) determinantal condition

$$
\left|1-G_{0} V\right|=0
$$

as written in an obvious notation.

For an explicit determination of the defect amplitude of vibration $\left|\chi^{2}\left(0, \omega^{2}\right)\right|$ in any given case of interest, one needs to introduce the lattice Green's functions of the impure crystal $(G)$ which are related to the above pure crystal lattice Green's functions $\left(G_{0}\right)$ via

$$
G=G_{0}+G_{0} V G_{0}+G_{0} V G_{0} V G_{0}+\ldots=\left(1-G_{0} V\right)^{-1} G_{0}
$$

As already noted above, the frequencies of the perturbed modes are given as the solutions to $\left|1-G_{0} V\right|=0$, while the intensities of interest in the impure and pure modes at the defect site (respectively $\left|\chi^{2}\left(0, \omega^{2}\right)\right|$ and $\left.\left|\chi_{\text {pure }}^{2}\left(0, \omega^{2}\right)\right|=1 / N M\right)$ are related by (see e.g. [6])

$$
\operatorname{Im} G_{x x}(0,0)=\frac{\left|\chi^{2}\left(0, \omega^{2}\right)\right|}{\left|\chi_{\text {pure }}^{2}\left(0, \omega^{2}\right)\right|} \operatorname{Im}\left(G_{0}\right)_{x x}(0,0)=\left|\chi^{2}\left(0, \omega^{2}\right)\right| \pi N \nu\left(\omega^{2}\right),
$$

where $\nu\left(\omega^{2}\right)$ is the density of squared frequency states of the pure crystal as normalized to one. It is the quantity $\operatorname{Im} G_{x x}(0,0)$ which determines the response of the system to an external probe, with the PDOS which gives the probability for nuclear resonant inelastic x-ray scattering at frequency $\omega$ being given by $D(E)=M^{\prime}\left|\chi^{2}\left(0, \omega^{2}\right)\right| \nu(\omega)=2 \omega M^{\prime}\left|\chi^{2}\left(0, \omega^{2}\right)\right| \nu\left(\omega^{2}\right)=$ $\left(2 \omega M^{\prime} / \pi N\right) \operatorname{Im} G_{x x}(0,0)$. The defect amplitude $\left|\chi^{2}\left(0, \omega^{2}\right)\right|$ modulates the response of the system and it is thus its determination which is needed for inelastic Mössbauer studies [9].

In an actual application of Eq. (17) to determine the needed $\left|\chi^{2}\left(0, \omega^{2}\right)\right|$, the key step is in inverting the matrix $1-G_{0} V$ as needed to obtain $G_{x x}(0,0)$ via Eq. (16). And even if one restricts to nearest neighbor force-constants only (which we will in fact do here and throughout) and to just a single substitutional defect, in the force-constant change case the matrix $V_{\alpha \beta}\left(\ell, \ell^{\prime}\right)$ will involve the defect and every single one of its host nearest neighbor atoms. For a simple cubic crystal for instance this defect-nearest neighbor complex has 21 degrees of freedom (seven 3-dimensional vibrations due to one defect and six neighbors), while for the body-centered and face-centered cubic crystals the complexes respectively have 27 and 39 relevant degrees of freedom, to initially make the matrix $V_{\alpha \beta}\left(\ell, \ell^{\prime}\right)$ and the non-trivial sector of the matrix $1-G_{0} V$ quite large. However, because of the high $O_{h}$ symmetry at the defect site the $V_{\alpha \beta}\left(\ell, \ell^{\prime}\right)$ matrix can be block diagonalized in the irreducible representations of the octahedral group, with the relevant decompositions in the simple, body-centered and face-centered cubic crystals being of the form (see e.g. [4])

$$
\begin{aligned}
\Gamma_{\mathrm{sc}} & =A_{1 g}+E_{g}+F_{1 g}+F_{2 g}+3 F_{1 u}+F_{2 u} \\
\Gamma_{\mathrm{bcc}} & =A_{1 g}+E_{g}+F_{1 g}+2 F_{2 g}+A_{2 u}+E_{u}+3 F_{1 u}+F_{2 u} \\
\Gamma_{\mathrm{fcc}} & =A_{1 g}+A_{2 g}+2 E_{g}+2 F_{1 g}+2 F_{2 g}+A_{2 u}+E_{u}+4 F_{1 u}+2 F_{2 u} .
\end{aligned}
$$

Then, since the displacement of the defect atom itself transforms as a 3-dimensional vector, the defect displacements must be located entirely within the $F_{1 u}$ modes, with all of 
the other irreducible representations being built out of displacements of appropriate linear combinations which involve the nearest neighbors of the defect alone. Since it is the defect response to which external probes such as nuclear resonant inelastic x-ray scattering couple, the sector of $V_{\alpha \beta}\left(\ell, \ell^{\prime}\right)$ which is relevant for such scattering thus reduces to respective 3-dimensional, 3-dimensional and 4-dimensional blocks in Eq. (16) each one of which is itself threefold degenerate. For the physically interesting body-centered cubic crystal the normalized 3-dimensional $3 F_{1 u}$ mode basis is given (in body-centered cubic crystal site notation) by

$$
\begin{aligned}
\alpha_{0}= & u_{x}(0,0,0), \\
\alpha_{1}= & \frac{1}{2 \sqrt{2}}\left[u_{x}(1,1,1)+u_{x}(\overline{1}, 1,1)+u_{x}(\overline{1}, \overline{1}, 1)+u_{x}(1, \overline{1}, 1)\right. \\
& \left.+u_{x}(\overline{1}, \overline{1}, \overline{1})+u_{x}(1, \overline{1}, \overline{1})+u_{x}(1,1, \overline{1})+u_{x}(\overline{1}, 1, \overline{1})\right], \\
\alpha_{2}= & \frac{1}{4}\left[u_{y}(1,1,1)+u_{z}(1,1,1)-u_{y}(\overline{1}, 1,1)-u_{z}(\overline{1}, 1,1)\right. \\
& +u_{y}(\overline{1}, \overline{1}, 1)-u_{z}(\overline{1}, \overline{1}, 1)-u_{y}(1, \overline{1}, 1)+u_{z}(1, \overline{1}, 1) \\
& +u_{y}(\overline{1}, \overline{1}, \overline{1})+u_{z}(\overline{1}, \overline{1}, \overline{1})-u_{y}(1, \overline{1}, \overline{1})-u_{z}(1, \overline{1}, \overline{1}) \\
& \left.+u_{y}(1,1, \overline{1})-u_{z}(1,1, \overline{1})-u_{y}(\overline{1}, 1, \overline{1})+u_{z}(\overline{1}, 1, \overline{1})\right],
\end{aligned}
$$

while for the equally interesting face-centered cubic crystal the normalized 4-dimensional $4 F_{1 u}$ mode basis is given (in face-centered cubic crystal site notation) by

$$
\begin{aligned}
\alpha_{0}= & u_{x}(0,0,0), \\
\alpha_{1}= & \frac{1}{2 \sqrt{2}}\left[u_{x}(1,1,0)+u_{x}(\overline{1}, \overline{1}, 0)+u_{x}(1,0,1)+u_{x}(\overline{1}, 0, \overline{1})\right. \\
& \left.+u_{x}(1, \overline{1}, 0)+u_{x}(\overline{1}, 1,0)+u_{x}(\overline{1}, 0,1)+u_{x}(1,0, \overline{1})\right], \\
\alpha_{2}= & \frac{1}{2 \sqrt{2}}\left[u_{y}(1,1,0)+u_{y}(\overline{1}, \overline{1}, 0)+u_{z}(1,0,1)+u_{z}(\overline{1}, 0, \overline{1})\right. \\
& \left.-u_{y}(1, \overline{1}, 0)-u_{y}(\overline{1}, 1,0)-u_{z}(\overline{1}, 0,1)-u_{z}(1,0, \overline{1})\right], \\
\alpha_{3}= & \frac{1}{2}\left[u_{x}(0,1,1)+u_{x}(0, \overline{1}, \overline{1})+u_{x}(0,1, \overline{1})+u_{x}(0, \overline{1}, 1)\right] .
\end{aligned}
$$

Degenerate with each of these bases are two others, one based on $u_{y}(0,0,0)$ and the other on $u_{z}(0,0,0)$. With the body-centered and face-centered cubic crystals being each other's reciprocal lattice, in the harmonic approximation where momentum and position are treated equivalently, the defect responses in the two cases must be identical [5], and it will thus suffice in the following to treat just one of the two of them. And with the body-centered cubic basis of Eq. (19) having lower dimensionality than the face-centered cubic crystal basis given in Eq. (20), in the following we shall treat the body-centered cubic alone. (While we do not treat the simple cubic crystal case here, for it one can anticipate results analogous to those we shall provide below for the body-centered cubic case.)

In terms of the basis of Eq. (19) the matrix of the pure crystal $G_{0}$ in the body-centered cubic $F_{1 u}$ mode can be written very compactly as [6] 


$$
\left[G_{0}\right]_{F_{1 u}}=\left(\begin{array}{ccc}
g_{0} & 2 \sqrt{ } 2 g_{1} & 4 g_{2} \\
2 \sqrt{ } 2 g_{1} & Q & \sqrt{ } 2 R \\
4 g_{2} & \sqrt{ } 2 R & S+T
\end{array}\right)
$$

where we have introduced the notation

$$
\begin{aligned}
& g_{0}=g_{x x}(000), \quad g_{1}=g_{x x}(111) \quad, \quad g_{2}=g_{x y}(111), \quad R=g_{x y}(222)+g_{x y}(220), \\
& Q=g_{0}+g_{x x}(222)+g_{x x}(200)+g_{x x}(022)+2 g_{x x}(220)+2 g_{x x}(020), \\
& S=g_{0}+g_{y y}(222)-g_{y y}(020)-g_{y y}(202) \quad, \quad T=g_{y z}(222)-g_{y z}(022)
\end{aligned}
$$

which takes advantage of the translational invariance of the pure crystal lattice to set $g_{\alpha \beta}\left(\omega ; \ell, \ell^{\prime}\right)=g_{\alpha \beta}\left(\omega ; \ell-\ell^{\prime}, 0\right)=g_{\alpha \beta}\left(\ell-\ell^{\prime}\right)$. The pure crystal Green's functions which appear in Eq. (21) are not completely independent of each other, as some of them are related via the general Eq. (13). Specifically, if Eq. (13) is restricted to nearest neighbor force-constants, for the body-centered cubic we obtain the relations [6]

$$
\begin{aligned}
& A_{x x}(0,0) g_{0}+8 A_{x x}(111) g_{1}+16 A_{x y}(111) g_{2}=1+M \omega^{2} g_{0}, \\
& 8 A_{x y}(111) g_{2}=0, \\
& A_{x x}(0,0) g_{1}+A_{x x}(111) Q+2 A_{x y}(111) R=M \omega^{2} g_{1}, \\
& A_{x x}(0,0) g_{2}+A_{x x}(111) R+A_{x y}(111)(S+T)=M \omega^{2} g_{2} .
\end{aligned}
$$

(Our notation here is to set $A_{\alpha \beta}\left(\ell, \ell^{\prime}\right)=A_{\alpha \beta}\left(\ell-\ell^{\prime}, 0\right)=A_{\alpha \beta}\left(\ell-\ell^{\prime}\right)$, but to use $A_{\alpha \beta}(0,0)$ to denote $A_{\alpha \beta}\left(\ell=0, \ell^{\prime}=0\right)$.) In establishing Eq. (23) we have used the fact that for the body-centered cubic crystal the lattice delta function $(1 / N) \sum_{\overrightarrow{\mathbf{k}}} e^{i \overrightarrow{\mathbf{k}} \cdot \overrightarrow{\mathbf{R}}\left(\ell^{\prime}\right)}$ with $\overrightarrow{\mathbf{R}}\left(\ell^{\prime}\right)=(p, q, r)$ has the property that it is equal to one if $p+q+r$ is an even integer and equal to zero otherwise [10]. As we see, via the use of Eq. (23) only two of the Green's functions which appear in Eq. (21) are independent. Evaluation of Eq. (16) can thus involve no more than two independent pure lattice Green's functions.

In order to be able characterize the various pure crystal force-constants $A_{\alpha \beta}\left(\ell, \ell^{\prime}\right)$ which appear in Eq. (23) as well as their impure $A_{\alpha \beta}^{\prime}\left(\ell, \ell^{\prime}\right)$ counterparts, we recall that in terms of the two-body host-host interatomic potential $\phi(r)$, the force-constants between a host atom vibrating around site $R_{\alpha}(\ell)$ and one vibrating around the origin are defined as

$$
A_{\alpha \beta}(\ell, 0)=-\left.\left(\frac{\partial^{2} \phi(r)}{\partial u_{\alpha}(\ell) \partial u_{\beta}(\ell)}\right)\right|_{0}=-\left(\frac{\phi^{\prime \prime}(r)}{r^{2}}-\frac{\phi^{\prime}(r)}{r^{3}}\right) R_{\alpha}(\ell) R_{\beta}(\ell)-\frac{\phi^{\prime}(r)}{r} \delta_{\alpha \beta},
$$

as calculated at the equilibrium lattice separation between the host atoms. Force-constants for which $\phi^{\prime}(r)$ just happens to vanish when the atoms are in their equilibrium positions are referred to as being central, while those associated with potentials which obey $\phi^{\prime}(r) / r=$ $\phi^{\prime \prime}(r)$ at equilibrium are referred to as being isotropic. In terms of the various $A_{\alpha \beta}(\ell, 0)$ with $\ell \neq 0$ the self-force-constant at the origin is then given via Newton's third law as the summation

$$
A_{\alpha \beta}(0,0)=-\sum_{\ell \neq 0} A_{\alpha \beta}(0, \ell)
$$

For a nearest neighbor pure crystal the force-constants can thus be characterized in terms of two parameters, viz. 


$$
\alpha=-\frac{1}{3}\left(\phi^{\prime \prime}(r)-\frac{\phi^{\prime}(r)}{r}\right), \beta=-\frac{\phi^{\prime}(r)}{r},
$$

in terms of which we obtain

$$
A_{\alpha \beta}(111)=\left(\begin{array}{ccc}
\alpha+\beta & \alpha & \alpha \\
\alpha & \alpha+\beta & \alpha \\
\alpha & \alpha & \alpha+\beta
\end{array}\right) \quad, \quad A_{\alpha \beta}(\overline{1} 11)=\left(\begin{array}{ccc}
\alpha+\beta & -\alpha & -\alpha \\
-\alpha & \alpha+\beta & \alpha \\
-\alpha & \alpha & \alpha+\beta
\end{array}\right)
$$

and

$$
A_{x x}(0,0)=-8(\alpha+\beta), A_{x y}(0,0)=0 .
$$

Given Eqs. (27) and (28) we can now rewrite the Green's function relations of Eq. (23) in the convenient form

$$
\begin{aligned}
8(\alpha+\beta) g_{1} & =8(\alpha+\beta) g_{0}+1+M \omega^{2} g_{0} \\
g_{2} & =0 \\
(\alpha+\beta) Q & =M \omega^{2} g_{1}+8(\alpha+\beta) g_{1}-2 \alpha R \\
\alpha(S+T) & =-(\alpha+\beta) R .
\end{aligned}
$$

When a defect is introduced substitutionally at a lattice site, the interatomic potential $\hat{\phi}(r)$ between it and a host neighbor will in principle differ from that between pairs of host atoms, leading to modified force-constants

$$
A_{\alpha \beta}^{\prime}(\ell, 0)=-\left.\left(\frac{\partial^{2} \hat{\phi}(r)}{\partial u_{\alpha}(\ell) \partial u_{\beta}(\ell)}\right)\right|_{0}=-\left(\frac{\hat{\phi}^{\prime \prime}(r)}{r^{2}}-\frac{\hat{\phi}^{\prime}(r)}{r^{3}}\right) R_{\alpha}(\ell) R_{\beta}(\ell)-\frac{\hat{\phi}^{\prime}(r)}{r} \delta_{\alpha \beta},
$$

and

$$
A_{\alpha \beta}^{\prime}(0,0)=-\sum_{\ell \neq 0} A_{\alpha \beta}^{\prime}(0, \ell)
$$

as again calculated at the host atom lattice equilibrium separation (the defect being inserted substitutionally), with the modified force-constants then being characterized by two parameters

$$
\hat{\alpha}=-\frac{1}{3}\left(\hat{\phi}^{\prime \prime}(r)-\frac{\hat{\phi}^{\prime}(r)}{r}\right) \quad, \quad \hat{\beta}=-\frac{\hat{\phi}^{\prime}(r)}{r},
$$

whose relation to $\alpha$ and $\beta$ can be arbitrary [11]. For the impure system we thus obtain

$$
A_{\alpha \beta}^{\prime}(111)=\left(\begin{array}{ccc}
\hat{\alpha}+\hat{\beta} & \hat{\alpha} & \hat{\alpha} \\
\hat{\alpha} & \hat{\alpha}+\hat{\beta} & \hat{\alpha} \\
\hat{\alpha} & \hat{\alpha} & \hat{\alpha}+\hat{\beta}
\end{array}\right), \quad A_{\alpha \beta}^{\prime}(\overline{1} 11)=\left(\begin{array}{ccc}
\hat{\alpha}+\hat{\beta} & -\hat{\alpha} & -\hat{\alpha} \\
-\hat{\alpha} & \hat{\alpha}+\hat{\beta} & \hat{\alpha} \\
-\hat{\alpha} & \hat{\alpha} & \hat{\alpha}+\hat{\beta}
\end{array}\right)
$$

and

$$
A_{x x}^{\prime}(0,0)=-8(\hat{\alpha}+\hat{\beta}), \quad A_{x y}^{\prime}(0,0)=0
$$


Finally, on introducing the parameters

$$
X=\alpha-\hat{\alpha}, \quad Y=\beta-\hat{\beta} .
$$

we can write the matrix elements of the perturbation $V_{\alpha \beta}\left(\ell, \ell^{\prime}\right)$ in the $F_{1 u}$ mode as

$$
[V]_{F_{1 u}}=\left(\begin{array}{ccc}
-\omega^{2}\left(M-M^{\prime}\right)-8 X-8 Y & 2 \sqrt{ } 2(X+Y) & 4 X \\
2 \sqrt{ } 2(X+Y) & -(X+Y) & -\sqrt{ } 2 X \\
4 X & -\sqrt{ } 2 X & -2 X-Y
\end{array}\right) .
$$

Armed with Eqs. (21), (29) and (36) we can now proceed to an evaluation of $\left(1-G_{0} V\right)^{-1} G_{0}$.

\section{SOLVING THE FORCE-CONSTANT CHANGE PROBLEM}

To solve the problem we first proceed symbolically and set

$$
\left[1-G_{0} V\right]_{F_{1 u}}=\left(\begin{array}{ccc}
a & b & c \\
d & e & f \\
g & h & i
\end{array}\right),
$$

where

$$
\begin{aligned}
a & =1-\omega^{2}\left(M^{\prime}-M\right) g_{0}+8(X+Y)\left(g_{0}-g_{1}\right), \\
b & =-2 \sqrt{ } 2(X+Y)\left(g_{0}-g_{1}\right), \\
c & =-4 X\left(g_{0}-g_{1}\right) \\
d & =-2 \sqrt{ } 2\left[\omega^{2}\left(M^{\prime}-M\right) g_{1}-(X+Y)\left(8 g_{1}-Q\right)+2 X R\right], \\
e & =1-(X+Y)\left(8 g_{1}-Q\right)+2 X R, \\
f & =\sqrt{ } 2\left[-X\left(8 g_{1}-Q\right)+(2 X+Y) R\right], \\
g & =-4(X+Y) R-4 X(S+T), \\
h & =\sqrt{ } 2[(X+Y) R+X(S+T)], \\
i & =1+2 X R+(2 X+Y)(S+T) .
\end{aligned}
$$

In terms of these symbolic quantities the inverse matrix is given by

$$
\left[1-G_{0} V\right]_{F_{1 u}}^{-1}=\frac{1}{\Delta}\left(\begin{array}{ccc}
e i-f h & h c-i b & f b-c e \\
f g-d i & i a-g c & c d-a f \\
d h-g e & g b-h a & a e-b d
\end{array}\right),
$$

where the determinant of $\left[1-G_{0} V\right]_{F_{1 u}}$ is given by

$$
\Delta=a(e i-f h)+d(h c-i b)+g(b f-c e) .
$$

From Eqs. (16) and (21) and recalling the vanishing of $g_{2}$, it then follows that the defect site component of the impure crystal Green's function $G$ is given in closed form by

$$
G_{x x}(0,0)=\frac{1}{\Delta}\left[(e i-f h) g_{0}+2 \sqrt{ } 2(h c-i b) g_{1}\right],
$$


with the matrix $1-G_{0} V$ having been inverted analytically.

For computational purposes we note that in both $\Delta$ and $G_{x x}(0,0)$ it is just three symbolic combinations, viz. $e i-f h, h c-i b$ and $b f-c e$, which are needed. From Eq. (38) these combinations are readily found to evaluate to

$$
\begin{aligned}
e i-f h= & 1+4 X R+(2 X+Y)(S+T)-(X+Y)\left(8 g_{1}-Q\right) \\
& +\left(3 X Y+Y^{2}\right)\left[-(S+T)\left(8 g_{1}-Q\right)-2 R^{2}\right], \\
h c-i b= & 2 \sqrt{ } 2\left(g_{0}-g_{1}\right)\left[X+Y+\left(3 X Y+Y^{2}\right)(S+T)\right], \\
b f-c e= & -4\left(g_{0}-g_{1}\right)\left[-X+\left(3 X Y+Y^{2}\right) R\right] .
\end{aligned}
$$

Further algebra shows that the numerator in Eq. (41) evaluates to

$$
\begin{aligned}
\mathrm{NUM}= & (e i-f h) g_{0}+2 \sqrt{ } 2(h c-i b) g_{1} \\
= & g_{0}[1+4 X R+(2 X+Y)(S+T)+(X+Y) Q] \\
& -8(X+Y) g_{1}^{2}+\left(3 X Y+Y^{2}\right)\left[(S+T)\left(g_{0} Q-8 g_{1}^{2}\right)-2 g_{0} R^{2}\right],
\end{aligned}
$$

while the denominator evaluates to

$$
\begin{aligned}
\Delta= & 1+4 X R+(X+Y)\left(8 g_{0}-16 g_{1}+Q\right)+(2 X+Y)(S+T) \\
& +\left(3 X Y+Y^{2}\right)\left[(S+T)\left(8 g_{0}-16 g_{1}+Q\right)-2 R^{2}\right]-8\left(M-M^{\prime}\right) \omega^{2}(X+Y) g_{1}^{2} \\
& +\left(M-M^{\prime}\right) \omega^{2} g_{0}[1+4 X R+(2 X+Y)(S+T)+(X+Y) Q] \\
& +\left(M-M^{\prime}\right) \omega^{2}\left(3 X Y+Y^{2}\right)\left[(S+T)\left(g_{0} Q-8 g_{1}^{2}\right)-2 g_{0} R^{2}\right] .
\end{aligned}
$$

To simplify the problem further we now utilize the pure crystal Green's function relations given in Eq. (29), to find, following a fair amount of algebra, that the numerator and denominator in $G_{x x}(0,0)$ reduce to

$$
\begin{aligned}
\mathrm{NUM}= & \frac{g_{0}}{\alpha(\alpha+\beta)}\left[\alpha(\alpha+\beta)-2 \beta^{2} X R-\left(3 \alpha^{2}+2 \alpha \beta+\beta^{2}\right) Y R\right] \\
& +\frac{g_{1}}{\alpha(\alpha+\beta)}\left[(\alpha+\beta)\left(3 X Y+Y^{2}\right) R-\alpha(X+Y)\right],
\end{aligned}
$$

and

$$
\begin{aligned}
\Delta= & \frac{\left[1+\left(M-M^{\prime}\right) \omega^{2} g_{0}\right]}{\alpha(\alpha+\beta)}\left[\alpha(\alpha+\beta)-2 \beta^{2} X R-\left(3 \alpha^{2}+2 \alpha \beta+\beta^{2}\right) Y R\right] \\
& +\frac{\left[1+M \omega^{2} g_{0}-M^{\prime} \omega^{2} g_{1}\right]}{\alpha(\alpha+\beta)}\left[(\alpha+\beta)\left(3 X Y+Y^{2}\right) R-\alpha(X+Y)\right],
\end{aligned}
$$

where

$$
g_{1}=g_{0}+\frac{\left[1+M \omega^{2} g_{0}\right]}{8(\alpha+\beta)} .
$$

In terms of the convenient functions

$$
\begin{aligned}
& \hat{R}=\frac{(\alpha+\beta)\left(3 X Y+Y^{2}\right) R}{\alpha(X+Y)}=\frac{(\alpha+\beta)(\beta-\hat{\beta})(3 \alpha-3 \hat{\alpha}+\beta-\hat{\beta}) R}{\alpha(\alpha-\hat{\alpha}+\beta-\hat{\beta})}, \\
& \mu=\frac{2(\beta X-\alpha Y)^{2}}{(\alpha+\beta)^{2}\left(3 X Y+Y^{2}\right)}=\frac{2(\alpha \hat{\beta}-\beta \hat{\alpha})^{2}}{(\alpha+\beta)^{2}(\beta-\hat{\beta})(3 \alpha-3 \hat{\alpha}+\beta-\hat{\beta})},
\end{aligned}
$$


which obey

$$
\mu \hat{R}=\frac{2(\alpha \hat{\beta}-\beta \hat{\alpha})^{2} R}{\alpha(\alpha+\beta)(\alpha-\hat{\alpha}+\beta-\hat{\beta})},
$$

the numerator and denominator in $G_{x x}(0,0)$ can be written more compactly as

$$
\begin{aligned}
\mathrm{NUM} & =\frac{A_{x x}^{\prime}(0,0)}{A_{x x}(0,0)}\left[g_{0}+\frac{\left(1+M \omega^{2} g_{0}\right)}{A_{x x}(0,0)}\left(\frac{A_{x x}(0,0)}{A_{x x}^{\prime}(0,0)}-1\right)\right][1-\hat{R}]-\mu g_{0} \hat{R} \\
& =\frac{1}{M \omega^{2}}\left[\frac{A_{x x}^{\prime}(0,0)}{A_{x x}(0,0)}\left(\rho\left(\omega^{2}\right) S\left(\omega^{2}\right)-\frac{M}{M^{\prime}} S\left(\omega^{2}\right)-1\right)[1-\hat{R}]+\mu\left[S\left(\omega^{2}\right)+1\right] \hat{R}\right],
\end{aligned}
$$

and

$$
\begin{aligned}
\Delta= & \frac{A_{x x}^{\prime}(0,0)}{A_{x x}(0,0)}\left\{1+M \omega^{2} g_{0}-M^{\prime} \omega^{2} g_{0}+\frac{M^{\prime} \omega^{2}}{A_{x x}(0,0)}-\frac{M^{\prime} \omega^{2}}{A_{x x}^{\prime}(0,0)}\right. \\
& \left.+\frac{M M^{\prime} \omega^{4} g_{0}}{A_{x x}(0,0)}-\frac{M M^{\prime} \omega^{4} g_{0}}{A_{x x}^{\prime}(0,0)}\right\}[1-\hat{R}]-\mu \hat{R}\left[1+\left(M-M^{\prime}\right) \omega^{2} g_{0}\right] \\
= & \frac{M^{\prime}}{M}\left\{\frac{A_{x x}^{\prime}(0,0)}{A_{x x}(0,0)}\left[1-\rho\left(\omega^{2}\right) S\left(\omega^{2}\right)\right][1-\hat{R}]-\mu \hat{R}\left[S\left(\omega^{2}\right)\left(1-\frac{M}{M^{\prime}}\right)+1\right]\right\}
\end{aligned}
$$

where $\rho\left(\omega^{2}\right)$ and $S\left(\omega^{2}\right)=-1-M \omega^{2} g_{0}$ were introduced earlier in Eqs. (2) and (3). The form for $G_{x x}(0,0)=\mathrm{NUM} / \Delta$ implied by Eqs. (50) and (51) is our main result, an exact relation for a body-centered cubic crystal under the sole assumption of nearest neighbor force-constants, with there being no restriction on the strengths of the central and noncentral force-constants in the pure crystal or the amount by which they might change in the presence of the defect, with the evaluation of $G_{x x}(0,0)$ requiring a knowledge of only two pure crystal lattice Green's functions, $g_{0}$ and $R$.

While the form of Eqs. (50) and (51) is completely general, great simplification occurs whenever we can set $\mu \hat{R}=0$, i.e. whenever the force-constant changes obey

$$
\alpha \hat{\beta}-\beta \hat{\alpha}=0
$$

since then $G_{x x}(0,0)$ reduces to

$$
G_{x x}(0,0)=\frac{\left[\rho\left(\omega^{2}\right) S\left(\omega^{2}\right)-\left(M / M^{\prime}\right) S\left(\omega^{2}\right)-1\right]}{M^{\prime} \omega^{2}\left[1-\rho\left(\omega^{2}\right) S\left(\omega^{2}\right)\right]},
$$

to then only depend on one pure lattice Green's function alone, the one at the defect site itself. In this restricted case we see that the positions of the eigenmodes are given as the solutions to

$$
1-\rho\left(\omega^{2}\right) S\left(\omega^{2}\right)=0
$$

while on giving $\omega^{2}$ a small imaginary part in the complex plane and recalling that

$$
\frac{1}{\left(\omega^{2}-\omega^{\prime 2}+i \epsilon\right)}=P\left(\frac{1}{\omega^{2}-\omega^{\prime 2}}\right)-i \pi \delta\left(\omega^{2}-\omega^{\prime 2}\right)
$$


find that the imaginary part of $G_{x x}(0,0)$ evaluates to

$$
\operatorname{Im} G_{x x}(0,0)=\frac{1}{M}\left(\frac{M}{M^{\prime}}\right)^{2}\left[\frac{\pi \nu\left(\omega^{2}\right)}{\left[1-\rho\left(\omega^{2}\right) S_{P}\left(\omega^{2}\right)\right]^{2}+\left[\pi \omega^{2} \nu\left(\omega^{2}\right) \rho\left(\omega^{2}\right)\right]^{2}}\right] .
$$

With the imaginary part of the pure crystal $g_{0}$ evaluating to $\operatorname{Im} g_{0}=\pi \nu\left(\omega^{2}\right) / M=$ $N\left|\chi_{\text {pure }}^{2}\left(0, \omega^{2}\right)\right| \pi \nu\left(\omega^{2}\right)$, Eqs. (1) and (4) thus follow [12]. With Eqs. (1) and (4) having previously been obtained under the central force-constant restriction, we see now that they in fact have far greater validity, since as well as the pure central force-constant solution to Eq. (52) in which $\beta=\hat{\beta}=0$, Eq. (52) also admits of other solutions. Specifically it admits of a pure isotropic force-constant solution in which $\alpha=\hat{\alpha}=0$, and also of solution in which the central and isotropic components of the force-constants undergo the same fractional change $\hat{\alpha} / \alpha=\hat{\beta} / \beta$.

While the family of solutions which obey $\alpha \hat{\beta}-\beta \hat{\alpha}=0$ encompasses a large and interesting class of force-constant changes, to go beyond this set of solutions requires including the second Green's function $R$. Unlike the Green's function $g_{0}$ whose evaluation depends only on the density of states of the pure crystal according to

$$
\begin{aligned}
g_{0} & =g_{x x}(\omega ; 0,0)=\frac{1}{3}\left[g_{x x}(\omega ; 0,0)+g_{y y}(\omega ; 0,0)+g_{z z}(\omega ; 0,0)\right] \\
& =\frac{1}{3 N M} \sum_{\overrightarrow{\mathbf{k}}, j} \frac{\left[\sigma_{x}^{* j}(\overrightarrow{\mathbf{k}}) \sigma_{x}^{j}(\overrightarrow{\mathbf{k}})+\sigma_{y}^{* j}(\overrightarrow{\mathbf{k}}) \sigma_{y}^{j}(\overrightarrow{\mathbf{k}})+\sigma_{z}^{* j}(\overrightarrow{\mathbf{k}}) \sigma_{z}^{j}(\overrightarrow{\mathbf{k}})\right]}{\left[\omega_{j}^{2}(\overrightarrow{\mathbf{k}})-\omega^{2}\right]} \\
& =\frac{1}{3 N M} \sum_{\overrightarrow{\mathbf{k}}, j} \frac{1}{\left[\omega_{j}^{2}(\overrightarrow{\mathbf{k}})-\omega^{2}\right]}=\frac{1}{M} \int_{0}^{\omega_{\max }^{2}} d \omega^{\prime 2} \frac{\nu\left(\omega^{\prime 2}\right)}{\left[\omega^{\prime 2}-\omega^{2}\right]},
\end{aligned}
$$

as can be seen from the definition of Eq. (12), any other pure lattice Green's function such as $R$ will involve a sum over momentum modes which cannot be reduced to an equivalent sum over eigenfrequencies. However, as noted in [5] it is possible to simply the evaluation of the lattice Green's functions which involve a non-zero $\overrightarrow{\mathbf{R}}\left(\ell^{\prime}\right)-\overrightarrow{\mathbf{R}}(\ell)$ to some degree, as it is possible to perform the sum over polarizations analytically. Specifically, it was shown that the general $g_{\alpha \beta}(\omega ; \ell, 0)$ with $\ell \neq 0$ can be written in closed form as

$$
g_{\alpha \beta}(\omega ; \ell, 0)=-\frac{1}{N M} \sum_{\overrightarrow{\mathbf{k}}} \lambda_{\alpha \beta}(\overrightarrow{\mathbf{k}}) e^{-i \overrightarrow{\mathbf{k}} \cdot \overrightarrow{\mathbf{R}}(\ell)}
$$

where the different components of $\lambda_{\alpha \beta}(\overrightarrow{\mathbf{k}})$ can be written in terms of the pure crystal dynamical matrix $D_{\alpha \beta}(\overrightarrow{\mathbf{k}})$ of Eq. (10) as

$$
\lambda_{x x}(\overrightarrow{\mathbf{k}})=\frac{\left[\omega^{4}-\omega^{2}\left(D_{y y}+D_{z z}\right)+D_{y y} D_{z z}-D_{y z}^{2}\right]}{\left[\omega^{6}-\omega^{4} \sum D_{x x}+\omega^{2}\left(\sum D_{y y} D_{z z}-\sum D_{x y}^{2}\right)-\prod D_{x x}+\sum D_{x x} D_{y z}^{2}-2 \prod D_{x y}\right]}
$$

and

$$
\lambda_{x y}(\overrightarrow{\mathbf{k}})=\frac{\left[\omega^{2} D_{x y}-D_{x y} D_{z z}+D_{x z} D_{y z}\right]}{\left[\omega^{6}-\omega^{4} \sum D_{x x}+\omega^{2}\left(\sum D_{y y} D_{z z}-\sum D_{x y}^{2}\right)-\prod D_{x x}+\sum D_{x x} D_{y z}^{2}-2 \prod D_{x y}\right]}
$$

(the sums being taken cyclically), to thus reduce a determination of the pure lattice Green's functions to a straightforward sum over the Brillouin zone. 


\section{ACKNOWLEDGMENTS}

The author would like to thank Dr. E. E. Alp for the kind hospitality of the Advanced Photon Source at Argonne National Laboratory where this work was performed. 


\section{REFERENCES}

[1] M. Seto, Y. Kobayashi, S. Kitao, R. Haruki, T. Mitsui, Y. Yoda, S. Nasu and S. Kikuta, Phys. Rev. B 61, 11420 (2000).

[2] M. Y. Hu, W. Sturhahn, T. S. Toellner, P. D. Mannheim, D. E. Brown, J. Zhao, and E. E. Alp, Phys. Rev. B 67, 094304 (2003).

[3] K. Parlinski, P. T. Jochym, O. Leupold, A. I. Chumakov, R. Rüffer, H. Schober, A. Jianu, J. Dutkiewicz and W. Maziarz, Phys. Rev. B 70, 224304 (2004).

[4] A. A. Maradudin, Rept. Prog. Phys. 28, 331 (1965).

[5] P. D. Mannheim, Phys. Rev. 165, 1011 (1968).

[6] P. D. Mannheim and S. S. Cohen, Phys. Rev. B 4, 3748 (1971).

[7] In Eq. (2) we have used the relation $\omega_{\max }^{2}=2 A_{x x}(0,0) / M$ which holds [5] for any pure harmonic cubic crystal in the nearest neighbor force-constant approximation. In Eq. (3) we have introduced the pure crystal lattice Green's function $g_{0}=g_{x x}(\omega ; 0,0)$ which we will describe below. The pure crystal density of squared frequencies $\nu\left(\omega^{\prime 2}\right)=\nu\left(\omega^{\prime}\right) / 2 \omega^{\prime}$ which is associated with $g_{0}$ is normalized to $\int_{0}^{\omega_{\max }^{2}} d \omega^{\prime 2} \nu\left(\omega^{\prime 2}\right)=1$.

[8] The PDOS is referred to as being partial since not all the nuclei in a given unit cell are necessarily Mössbauer active.

[9] In general for a perturbation $Q$ the response function is given by $K(\omega)=\omega \operatorname{Im} \operatorname{Tr} G Q$ where the trace is taken over the relevant $O_{h}$ representations.

[10] The four relations given in Eq. (23) respectively follow by setting $\left(\ell^{\prime}, \alpha, \alpha^{\prime}\right)$ equal to $(0, x, x),(0, x, y),(111, x, x)$ and $(111, x, y)$ in Eq. (13), restricting the sum on $\ell$ to the origin and its eight first neighbors, and using the symmetry relations $A_{x y}(0,0)=$ $0, A_{x x}(111)=A_{x x}(\overline{1} 11), A_{x y}(111)=-A_{x y}(\overline{1} 11), g_{x y}(0,0)=0, g_{x x}(111)=g_{x x}(\overline{1} 11)$, $g_{x y}(111)=-g_{x y}(\overline{111}), g_{x y}(002)=g_{x y}(020)=g_{x y}(200)=g_{x y}(022)=g_{x y}(202)=0$. In passing we note that in Ref. [6] the $8 A_{x y}(111) g_{2}=0$ relation (a relation which entails that the pure body-centered cubic lattice Green's function $g_{2}$ is actually zero in the nearest neighbor approximation) was not considered as it was not needed there, with the conclusions reached in the pure central force-constant study of $[5,6]$ not in any way being dependent on the value of $g_{2}$ since the use of the three other Green's functions relations given in Eq. (23) still allowed for its elimination in favor of the defect site $g_{0}$ anyway. However, for the simplification of the non-central force-constant calculations which are presented in this paper, the vanishing of $g_{2}$ turns out to be quite crucial.

[11] Even if the pure crystal lattice separation is such that nearest neighbor atoms just happen to be at the same distance as the minimum in the two body host-host interatomic potential (central force-constant crystal case), it does not follow that the minimum in the two body host-defect interatomic potential would occur at precisely that same distance.

[12] In passing we note that there is a difference between the usage of the real $\omega^{2}$ equation $1-\rho\left(\omega^{2}\right) S\left(\omega^{2}\right)=0$ of Eq. (54) which gives all the modes of the impure crystal and the usage of the complex $\omega^{2}$ condition $1-\rho\left(\omega^{2}\right) S_{P}\left(\omega^{2}\right)=0$ at which $\operatorname{Im} G_{x x}(0,0)$ of Eq. (56) is resonant, with there being at best only a handful of frequencies for which this resonant condition is satisfied, and possibly none at all if $\rho\left(\omega^{2}\right)$ is such that the $1-\rho\left(\omega^{2}\right) S\left(\omega^{2}\right)=0$ equation has a localized mode solution outside the pure crystal frequency band. (Outside the band there is no need to distinguish between $S\left(\omega^{2}\right)$ and $S_{P}\left(\omega^{2}\right)$ since $S\left(\omega^{2}\right)$ has no poles there.) Specifically, in applying Eq. (54) to identify all of the allowed impure crystal eigenmodes one uses the dis- 
crete form of $S\left(\omega^{2}\right)$ given according to Eqs. (3) and (12) and Eq. (57) below as $S\left(\omega^{2}\right)=-1-M \omega^{2} g_{x x}(\omega ; 0,0)=-(1 / N) \sum_{\overrightarrow{\mathbf{k}}, j}\left[\sigma_{x}^{* j}(\overrightarrow{\mathbf{k}}) \sigma_{x}^{j}(\overrightarrow{\mathbf{k}}) \omega_{j}^{2}(\overrightarrow{\mathbf{k}})\right] /\left[\omega_{j}^{2}(\overrightarrow{\mathbf{k}})-\omega^{2}\right]$, to find that the modes of the impure crystal lie either between the modes of the pure crystal where $S\left(\omega^{2}\right)$ diverges, or below the lowest pure crystal frequency mode or beyond the largest pure crystal frequency mode, with the discretized spectra of the pure and impure crystals having no common eigenfrequency. (Despite being a sum on both $\overrightarrow{\mathbf{k}}$ and $j$, the $\sigma_{x}^{* j}(\overrightarrow{\mathbf{k}}) \sigma_{x}^{j}(\overrightarrow{\mathbf{k}})$ term in the expression for $S\left(\omega^{2}\right)$ only permits the $j$ sum to contribute once, since in the basis in which $D_{\alpha \beta}(\overrightarrow{\mathbf{k}})$ is diagonalized we can choose the polarization vectors $\sigma_{x}^{j}(\overrightarrow{\mathbf{k}})$ so that only one polarization eigenvector has a non-zero $x$ component. The function $S\left(\omega^{2}\right)$ thus only possesses $N$ poles, with the full $3 N$ dimensionality of the eigenfrequencies then being recovered via the additional eigenmode equations for $u_{y}(0,0,0)$ and $u_{z}(0,0,0)$.) This lack of any common eigenfrequency between the pure and impure systems holds no matter what values one uses for the $M / M^{\prime}$ and $A_{x x}(0,0) / A_{x x}^{\prime}(0,0)$ parameters which appear in the $1-\rho\left(\omega^{2}\right) S\left(\omega^{2}\right)=0$ condition (H. J. Lipkin and P. D. Mannheim, Bounds on Localized Modes in the Crystal Impurity Problem, condmat/0510542), with the threefold degenerate $1-\rho\left(\omega^{2}\right) S\left(\omega^{2}\right)=0$ condition always generating a total of $3 N$ perturbed crystal eigenfrequencies. For perturbations which obey the $\alpha \hat{\beta}-\beta \hat{\alpha}=0$ condition of Eq. (52) then, the threefold degenerate $F_{1 u}$ sector condition $1-\rho\left(\omega^{2}\right) S\left(\omega^{2}\right)=0$ generates the full set of $3 N$ impure crystal eigenmodes, with there being no possibility of any further modes being generated via eigenvalue conditions associated with any of the other irreducible $O_{h}$ representations that are given in Eq. (18). 